\title{
Simultaneous $\mathcal{H}_{\infty}$ Stabilization via Fixed-Order Controllers: Equivalence and Computation
}

\author{
Zhan Shu James Lam Ping Li
}

\begin{abstract}
This paper is concerned with simultaneous $\mathcal{H}_{\infty}$ stabilization with a fixed-order constraint on the controllers for multi-input/multi-output (MIMO) plants. By virtue of a new closed-loop stability and $\mathcal{H}_{\infty}$ performance characterization, a novel necessary and sufficient condition for the existence of desired controllers is established in terms of a set of nonlinear matrix inequalities, which possess a monotonic structure for a linearized computation. A convergent iterative algorithm is then provided to solve the condition, and a special property of the feasible solutions enables one to further improve the solvability via simple optimization on initial values. In addition, structural constraints on the controller gains or strong stabilizability can be incorporated into the design procedure in a straightforward manner.
\end{abstract}

Index Terms-Fixed-order controller, $\mathcal{H}_{\infty}$ control, iterative calculation, linear matrix inequality (LMI), simultaneous $\mathcal{H}_{\infty}$ stabilization, structural gains, strong stabilization.

\section{INTRODUCTION}

Over decades, a great deal of effort has been devoted to studying the simultaneous stabilization problem formulated initially in [1] and [2]. Simultaneous stabilization has been shown to be relevant to several important systems and control problems including strong stabilization [3], model reduction [4], and robust stabilization [5], and has found widespread practical applications such as nonlinear control for helicopters [6], chemical process control [7], faulttolerant control [8].

Early investigations on simultaneous stabilization are available in [9], [10], [11] and reference therein. A significant contribution to this problem comes from Blondel's research [12], [13], [14], which provide some fundamental results from the theory of computational complexity. It has been shown that the computational complexity of the simultaneous stabilization problem with a fixed-order controller is NPhard [12], [13], that is, it is very unlikely to be solved via polynomial time algorithms, and even without a constraint on the controller order, it is not rationally decidable as well [14]. Although the simultaneous stabilization problem in its general form seems to be numerically intractable, it is still possible to partially/approximately solve it via restricting the class of plants or resorting to efficient computational

Zhan Shu (hustd8 @yahoo.cn) was previously with the Department of Mechanical Engineering, The University of Hong Kong, and is now with the Hamilton Institute, National University of Ireland, Maynooth, Co. Kildare, Ireland. His research was partially supported by Science Foundation of Ireland, Grant 07/PI/I1838.

James Lam (james. lam@hku.hk) and Ping Li are with the Department of Mechanical Engineering, The University of Hong Kong, Pokfulam Road, Hong Kong. Their research was partially supported by GRF HKU $7137 / 09 \mathrm{E}$ approaches. For the single-input single-output case, a rankone LMI approach to fixed-order simultaneous stabilization has been developed in [15]. For the multi-input multioutput case with a common output matrix, novel matrix inequality characterizations for static state/output-feedback simultaneous stabilizability and corresponding iterative LMI algorithm have been established in [16]. When disturbance attenuation is concerned, a linear periodically time-varying control scheme, under some stringent constraints on the plants, has been proposed in [17] to achieve near-optimal $\mathcal{H}_{\infty}$ performance. Recently, some effort in [18] has been devoted to simultaneous stabilization with stable controllers under the chain scattering framework, however, the controller order is generally not guaranteed.

The focus of the paper is simultaneous $\mathcal{H}_{\infty}$ stabilization via fixed-order controllers. From a theoretical point of view, a novel necessary and sufficient condition for the existence of desired controllers is established based on a new closedloop stability and $\mathcal{H}_{\infty}$ performance characterization. In the new condition, the controller gain is parametrized by a common free positive definite matrix independent of the multiple Lyapunov matrices, and the arbitrariness of the matrix enables one to incorporate additional design specifications such as structural gains or strong stabilizability into the design procedure readily. From a numerical point of view, the obtained design condition possesses a monotonic structure, which leads to a simple linearized computation in an iterative way, and a special property of the feasible solutions paves the way to improve the solvability via some optimization techniques. The analysis and synthesis are carried out in the time-domain, and thus all the results may be extended to other types of systems, for example, stochastic systems, switched systems, or even nonlinear systems, in a straightforward manner.

Notation: Throughout this paper, for real symmetric matrices $X$ and $Y$, the notation $X \geq Y$ (respectively, $X>$ $Y$ ) means that the matrix $X-Y$ is positive semidefinite (respectively, positive definite); $I$ is the identity matrix with appropriate dimension; $0_{n}$ and $I_{n}$ represent the $n$ by $n$ zero matrix, and the $n$ order identity matrix, respectively; The superscript " $T$ " represents the transpose; $\|\cdot\|$ denotes Euclidean norm for vectors or the spectral norm for matrices; $\operatorname{diag}\left(A_{1}, A_{2}, \ldots, A_{N}\right)$ denotes the block diagonal matrix composed by matrices $A_{i}, i=1,2, \ldots, N$; For a matrix $A \in \mathbb{R}^{n \times n}, \operatorname{Herm}(A)=A+A^{T}$; For a transfer function matrix $G(s),\|G\|_{\infty}$ represents the $\mathcal{H}_{\infty}$ norm of $G(s)$; For a matrix $C \in \mathbb{R}^{m \times n}, C^{\perp}$ denotes the orthogonal complement of $C$; The symbol \# is used to denote a matrix which can be 
inferred by symmetry; Matrices, if their dimensions are not explicitly stated, are assumed to have compatible dimensions for algebraic operations.

\section{PRELIMINARIES AND PROBLEM FORMULATION}

Consider the following family of linear plants

$$
\left\{\begin{array}{l}
\dot{x}_{i}(t)=A_{i} x_{i}(t)+B_{i} u_{i}(t)+B_{w i} w_{i}(t), \\
z_{i}(t)=C_{i} x_{i}(t)+D_{i} u_{i}(t)+D_{w i} w_{i}(t), \\
y_{i}(t)=C_{y i} x_{i}(t)+D_{y w i} w_{i}(t),
\end{array}\right.
$$

with $i \in \mathbb{V}=\{1,2, \ldots, r\}$, where $x_{i}(t) \in \mathbb{R}^{n}, u_{i}(t) \in \mathbb{R}^{n_{u}}$, $y_{i}(t) \in \mathbb{R}^{n_{y}}, z_{i}(t) \in \mathbb{R}^{n_{z}}$, and $w_{i}(t) \in \mathbb{R}^{n_{w}}$ are the system state, the control input, the measured output, the regulated output to be controlled, and the exogenous disturbance input, respectively. The controller under consideration is of the form

$$
\mathcal{U}:\left\{\begin{array}{l}
\dot{\xi}(t)=K_{A} \xi(t)+K_{B} y(t), \\
u(t)=K_{C} \xi(t)+K_{D} y(t),
\end{array}\right.
$$

where $K_{A} \in \mathbb{R}^{n_{c} \times n_{c}}, K_{B} \in \mathbb{R}^{n_{c} \times n_{y}}, K_{C} \in \mathbb{R}^{n_{u} \times n_{c}}$, and $K_{D} \in \mathbb{R}^{n_{u} \times n_{y}}$ are the controller matrices to be designed. When the controller in (2) is applied to the family of plants in (1), the closed-loop family of plants becomes

$$
\mathcal{T}:\left\{\begin{aligned}
\dot{x}_{c l i}(t) & =A_{c l i} x_{c l i}(t)+B_{c l w i} w_{i}(t), \\
z_{i}(t) & =C_{c l i} x_{c l i}(t)+D_{c l w i} w_{i}(t),
\end{aligned}\right.
$$

where $x_{c l i}(t)=\left[\begin{array}{ll}x_{i}^{T}(t) & \xi^{T}(t)\end{array}\right]^{T}$,

$$
\begin{aligned}
A_{c l i} & =\bar{A}_{i}+\bar{B}_{i} K \bar{C}_{y i}, & B_{c l w i} & =\bar{B}_{w i}+\bar{B}_{i} K \bar{D}_{y w i}, \\
C_{c l i} & =\bar{C}_{i}+\bar{D}_{i} K \bar{C}_{y i}, & D_{c l w i} & =\bar{D}_{w i}+\bar{D}_{i} K \bar{D}_{y w i},
\end{aligned}
$$

and

$$
\begin{array}{rlrl}
\bar{A}_{i} & =\left[\begin{array}{cc}
A_{i} & 0 \\
0 & 0
\end{array}\right], & \bar{B}_{i}=\left[\begin{array}{cc}
0 & B_{i} \\
I & 0
\end{array}\right], & \bar{B}_{w i}=\left[\begin{array}{c}
B_{w i} \\
0
\end{array}\right], \\
\bar{C}_{i}=\left[\begin{array}{ll}
C_{i} & 0
\end{array}\right], & \bar{D}_{i}=\left[\begin{array}{cc}
0 & D_{i}
\end{array}\right], & \bar{D}_{w i}=D_{w i}, \\
K=\left[\begin{array}{cc}
K_{A} & K_{B} \\
K_{C} & K_{D}
\end{array}\right], & \bar{C}_{y i}=\left[\begin{array}{cc}
0 & I \\
C_{y i} & 0
\end{array}\right], & \bar{D}_{y w i}=\left[\begin{array}{c}
0 \\
D_{y w i}
\end{array}\right] .
\end{array}
$$

The simultaneous $\mathcal{H}_{\infty}$ stabilization (SHS) problem to be solved in this paper is addressed as follows.

Problem 1 (SHS): Design a controller in (2) such that every closed-loop system family in (3) is asymptotically stable and satisfies

$$
\left\|\mathcal{T}_{z_{i} w_{i}}\right\|_{\infty}<\gamma_{i}, \quad i \in \mathbb{V},
$$

where $\mathcal{T}_{z_{i} w_{i}}(s)$ represents the transfer function matrix of the $i$ th system of (3) from $w_{i}$ to $z_{i}$.

A controller in (2) is said to be a structural solution to Problem SHS if it solves Problem SHS, and has some prescribed structural constraints on the controller matrix $K$. For example, block diagonal, upper (lower) triangular, sparse, or other prescribed patterns. Furthermore, a controller in (2) is said to be a $\gamma_{K}$-stable solution to Problem SHS if it solves Problem SHS and satisfies

$$
\mathcal{U}_{u y}(s) \text { is stable, and }\left\|\mathcal{U}_{u y}\right\|_{\infty}<\gamma_{K},
$$

where $\mathcal{U}_{u y}(s)$ is the transfer function matrix of (2) from $y$ to $u$. We end this section by giving the following elimination lemma, which will be used in the sequel.

Lemma 1 ([19]): Let $G \in \mathbb{R}^{n \times n}$ be a real symmetric matrix and $U, V$ be real matrices with appropriate dimensions. Then,

$$
G+U^{T} X V+V^{T} X^{T} U>0
$$

holds for some $X$ if and only if

$$
\left(V^{\perp}\right)^{T} G V^{\perp}>0,\left(U^{\perp}\right)^{T} G U^{\perp}>0 .
$$

Note that if $V$ or $U$ has rank $n$, then the first or second inequality in (4) will disappear.

\section{MAIN RESULTS}

\section{A. New Closed-Loop Stability and $\mathcal{H}_{\infty}$ Performance Char- acterization}

Theorem 1: The following statements are equivalent:

1) Every closed-loop system family in (3) is asymptotically stable and satisfies $\left\|\mathcal{T}_{z_{i} w_{i}}\right\|_{\infty}<\gamma_{i}$.

2) There exist $P_{i}>0, i \in \mathbb{V}$, an arbitrary $S>0$, and a sufficiently large scalar $\alpha>0$ such that, for each $i \in \mathbb{V}$,

$$
\Omega_{i}=\left[\begin{array}{cc}
\operatorname{Herm}\left(\mathcal{P}_{i}^{T}(\alpha) \mathcal{A}_{i}\right)+\Lambda_{i} & \# \\
\mathcal{C}_{i} & -\gamma_{i} I
\end{array}\right]<0,
$$

where

$$
\begin{aligned}
\mathcal{P}_{i}(\alpha) & =\left[\begin{array}{ccc}
P_{i} & 0 & 0 \\
-\alpha S K \bar{C}_{y i} & \alpha S & 0 \\
P_{i} & 0 & 0 \\
-\alpha S K \bar{C}_{y i} & \alpha S & -\alpha S K \bar{D}_{y w i}
\end{array}\right], \\
\mathcal{A}_{i} & =\left[\begin{array}{ccc}
\bar{A}_{i} & \bar{B}_{i} & \bar{B}_{w i} \\
K \bar{C}_{y i} & -I & K \bar{D}_{y w i} \\
0 & 0 & 0 \\
0 & 0 & K \bar{D}_{y w i}
\end{array}\right] \\
\Lambda_{i} & =\left[\begin{array}{ccc}
0_{n+n_{c}} & 0 & 0 \\
0 & 0_{n_{u}+n_{c}} & 0 \\
0 & 0 & -\gamma_{i} I_{n_{w}}
\end{array}\right] \\
\mathcal{C}_{i} & =\left[\begin{array}{lll}
\bar{C}_{i} & \bar{D}_{i} & \bar{D}_{w i}
\end{array}\right] .
\end{aligned}
$$

3) There exist $P_{i}>0, i \in \mathbb{V}$, an arbitrary $S>0$, a sufficiently large scalar $\alpha>0$ such that, for each $i \in$ $\mathbb{V}$,

$$
\left(\mathcal{Z}_{i}^{\perp}\right)^{T}\left[\begin{array}{ccc}
\Psi_{1 i}(\alpha) & \# & \# \\
\Psi_{2 i} & -2 \mathcal{S}_{+}(\alpha) & \# \\
\mathcal{C}_{i} & 0 & -\gamma_{i} I
\end{array}\right] \mathcal{Z}_{i}^{\perp}<0
$$

where

$$
\begin{aligned}
\Psi_{1 i}(\alpha) & =\operatorname{Herm}\left(\left(\mathcal{P}_{i}(\alpha)-\mathcal{R}_{i}\right)^{T} \mathcal{A}_{i}\right)+\Lambda_{i}, \\
\Psi_{2 i} & =\mathcal{R}_{i}+\mathcal{S A}_{i}
\end{aligned}
$$




$$
\begin{aligned}
\mathcal{S} & =\operatorname{diag}(0, S, 0, S), \\
\mathcal{S}_{+}(\alpha) & =\operatorname{diag}(0, S, 0, \alpha S), \\
\mathcal{R}_{i} & =\left[\begin{array}{ccc}
P_{i} & 0 & 0 \\
0 & 0 & 0 \\
P_{i} & 0 & 0 \\
0 & 0 & 0
\end{array}\right], \\
\mathcal{Z}_{i} & =\left[\begin{array}{cccccccc}
\bar{A}_{i} & \bar{B}_{i} & \bar{B}_{w i} & -I & 0 & 0 & 0 & 0 \\
0 & 0 & 0 & 0 & 0 & -I & 0 & 0
\end{array}\right] .
\end{aligned}
$$

Proof: 3) $\Rightarrow 2$ ) It follows from (6) and Lemma 1 that there exist matrices $\Delta_{i}$ such that

$$
\left[\begin{array}{ccc}
\Psi_{1 i}(\alpha) & \# & \# \\
\Psi_{2 i} & -2 \mathcal{S}_{+}(\alpha) & \# \\
\mathcal{C}_{i} & 0 & -\gamma_{i} I
\end{array}\right]+\operatorname{Herm}\left(\mathcal{Z}_{i}^{T} \Delta_{i}\right)<0
$$

Pre- and post-multiplying (7) by $\mathcal{Y}_{i} \mathcal{I}(\alpha)$ and its transpose, where

$$
\begin{aligned}
\mathcal{Y}_{i}= & {\left[\begin{array}{ccc}
I_{2 n_{c}+n_{u}+n+n_{w}} & \mathcal{A}_{i}^{T} & 0 \\
0 & 0 & I_{n_{z}}
\end{array}\right], } \\
\mathcal{I}(\alpha)= & \operatorname{diag}\left(I_{n+n_{c}}, I_{n_{c}+n_{u}}, I_{n_{w}}, I_{n+n_{c}}, I_{n_{c}+n_{u}},\right. \\
& \left.I_{n+n_{c}}, \alpha^{-1} I_{n_{c}+n_{u}}, I_{n_{z}}\right),
\end{aligned}
$$

and noticing that $\mathcal{Y}_{i} \mathcal{I}(\alpha) \mathcal{Z}_{i}^{T}=0$, one has that (5) holds.

$2) \Rightarrow 1$ ) Define a nonsingular transformation matrix as follows:

$$
T_{i}=\left[\begin{array}{cccc}
I & 0 & 0 & 0 \\
K \bar{C}_{y i} & K \bar{D}_{y w i} & 0 & I \\
0 & I & 0 & 0 \\
0 & 0 & I & 0
\end{array}\right] .
$$

Pre- and post-multiplying (5) by $T_{i}^{T}$ and $T_{i}$, respectively, yield that

$$
\begin{aligned}
& T_{i}^{T} \Omega_{i} T_{i} \\
= & {\left[\begin{array}{cccc}
P_{i} A_{c l i}+A_{c l i}^{T} P_{i} & \# & \# & \# \\
B_{c l w i}^{T} P_{i} & -\gamma_{i} I & \# & \# \\
C_{c l i} & D_{c l w i} & -\gamma_{i} I & \# \\
\bar{B}_{i}^{T} P_{i} & 0 & \bar{D}_{i}^{T} & -2 \alpha S
\end{array}\right] } \\
< & 0,
\end{aligned}
$$

of which the 3rd leading principal submatrix implies that every closed-loop system family in (3) is asymptotically stable and satisfies $\left\|\mathcal{T}_{z_{i} w_{i}}\right\|_{\infty}<\gamma_{i}$ (see bounded real lemma [20]).

1) $\Rightarrow 3)$ 1) implies that there exist $P_{i}>0, i \in \mathbb{V}$, such that, for each $i \in \mathbb{V}$,

$$
\mathrm{BRL}_{i} \triangleq\left[\begin{array}{ccc}
P_{i} A_{c l i}+A_{c l i}^{T} P_{i} & P_{i} B_{c l w i} & C_{c l i}^{T} \\
B_{c l w i}^{T} P_{i} & -\gamma_{i} I & D_{c l w i}^{T} \\
C_{c l i} & D_{c l w i} & -\gamma_{i} I
\end{array}\right]<0 .
$$

Let $S>0$ be arbitrary, and

$$
\begin{aligned}
\Delta_{i} & \triangleq\left[\begin{array}{cccccccc}
P_{i} & 0 & 0 & \varepsilon I & 0 & 0 & 0 & 0 \\
P_{i} & 0 & 0 & 0 & 0 & \varepsilon I & 0 & 0
\end{array}\right], \\
\mathcal{E} & \triangleq \operatorname{diag}(\varepsilon I, 0, \varepsilon I, 0),
\end{aligned}
$$

where the partitions are compatible with $\mathcal{Z}_{i}$ and $\mathcal{S}(\alpha)$, respectively, and $\varepsilon>0$ is a sufficiently small scalar such that, for each $i \in \mathbb{V}$,

$$
\mathrm{BRL}_{i}+\frac{1}{2} \varepsilon\left[\begin{array}{c}
A_{c l i}^{T} \\
B_{c l w i}^{T} \\
0
\end{array}\right]\left[\begin{array}{c}
A_{c l i}^{T} \\
B_{c l w i}^{T} \\
0
\end{array}\right]^{T}<0 .
$$

Now, set $\alpha_{1}>0$ and $\alpha_{2}>0$ to be sufficiently large scalars such that, for each $i \in \mathbb{V}$,

$$
\begin{aligned}
\Xi_{i}\left(\alpha_{1}\right) \triangleq & \mathrm{BRL}_{i}+\frac{1}{2} \varepsilon\left[\begin{array}{c}
A_{c l i}^{T} \\
B_{c l w i}^{T} \\
0
\end{array}\right]\left[\begin{array}{c}
A_{c l i}^{T} \\
B_{c l w i}^{T} \\
0
\end{array}\right]^{T} \\
& +\frac{1}{2 \alpha_{1}}\left[\begin{array}{c}
0 \\
\bar{D}_{y w i}^{T} \\
0
\end{array}\right] K^{T} S K\left[\begin{array}{c}
0 \\
\bar{D}_{y w i}^{T} \\
0
\end{array}\right]^{T} \\
< & 0,
\end{aligned}
$$

and

$$
-\Sigma_{i} \Xi_{i}^{-1}\left(\alpha_{1}\right) \Sigma_{i}^{T}+\frac{1}{2} \varepsilon \bar{B}_{i}^{T} \bar{B}_{i}+\frac{1}{2}\left(1-4 \alpha_{2}\right) S<0,
$$

where $\Sigma_{i}=\left[\begin{array}{lll}\bar{B}_{i}^{T} P_{i}+\frac{1}{2} \varepsilon \bar{B}_{i}^{T} A_{c l i} & \frac{1}{2} \varepsilon \bar{B}_{i}^{T} B_{\text {clwi }} & \bar{D}_{i}^{T}\end{array}\right]$. For $\alpha=\max \left\{\alpha_{1}, \alpha_{2}\right\}$, it is easy to verify that

$$
-\Sigma_{i} \Xi_{i}^{-1}(\alpha) \Sigma_{i}^{T}+\frac{1}{2} \varepsilon \bar{B}_{i}^{T} \bar{B}_{i}+\frac{1}{2}(1-4 \alpha) S<0,
$$

Then, by (10) and Schur complement equivalence [19], one obtains that

$$
\begin{aligned}
& T_{i}^{T}\left(\Omega_{i}+\frac{1}{2}\left[\begin{array}{cc}
\mathcal{A}_{i}^{T}\left(\mathcal{S}_{-}(\alpha)+\mathcal{E}\right) \mathcal{A}_{i} & 0 \\
0 & 0
\end{array}\right]\right) T_{i} \\
= & {\left[\begin{array}{cc}
\Xi_{i}(\alpha) & \# \\
\Sigma_{i} & \frac{1}{2} \varepsilon \bar{B}_{i}^{T} \bar{B}_{i}+\frac{1}{2}(1-4 \alpha) S
\end{array}\right] } \\
< & 0,
\end{aligned}
$$

where $\mathcal{S}_{-}(\alpha)=\operatorname{diag}\left(0, S, 0, \alpha^{-1} S\right)$. It follows from (11) and Schur complement equivalence that

$$
\begin{aligned}
& {\left[\begin{array}{ccc}
\Psi_{1 i}(\alpha) & \# & \# \\
\Psi_{2 i} & -2 \mathcal{S}_{+}(\alpha) & \# \\
\mathcal{C}_{i} & 0 & -\gamma_{i} I
\end{array}\right]+\operatorname{Herm}\left(\mathcal{Z}_{i}^{T} \Delta_{i}\right)} \\
& =\mathcal{I}^{-T}(\alpha)\left[\begin{array}{c}
\operatorname{Herm}\left(\mathcal{P}_{i}^{T}(\alpha) \mathcal{A}_{i}\right)+\Lambda_{i} \\
\left(\mathcal{S}_{-}(\alpha)+\mathcal{E}\right) \mathcal{A}_{i} \\
\mathcal{C}_{i}
\end{array}\right. \\
& \left.\begin{array}{cc}
\# & \# \\
-2\left(\mathcal{S}_{-}(\alpha)+\mathcal{E}\right) & \# \\
0 & -\gamma_{i} I
\end{array}\right] \mathcal{I}^{-1}(\alpha) \\
& <0 \text {, }
\end{aligned}
$$

which by Lemma 1 implies that (6) holds. This completes the proof.

Remark 1: In most previous LMI formulations, the Lyapunov matrix used for checking stability or performances is coupled with the controller matrix. This may induce additional constraints on the Lyapunov matrix when the controller matrix is parametrized. The significance of the conditions in Theorem 1 lies in the separation of the multiple Lyapunov matrices $P_{i}$ and the single controller matrix $K$, which avoids imposing any constraint on $P_{i}$ when $K$ is parametrized, and the introduction of a common matrix $S$ for the parametrization of $K$. 
Remark 2: The arbitrariness of $S$ enables one to impose additional constraints on the controller matrix without loss of generality, and thus various synthesis problems, such as structural controller synthesis or strong stabilization, can be treated readily under the same framework.

Remark 3: Although conditions 2) and 3) are equivalent, whereas, 3) may be more desirable from a computational point of view. This is because the multipliers $\Delta_{i}$, also referred to as slack matrix variables in some cases, have been introduced in 3), and they are expected to make the iteration to be presented later more efficient and less conservative.

\section{B. Design Condition and Algorithm}

We are now in a position to establish a new necessary and sufficient condition for the existence of the desired controllers.

Theorem 2: Problem SHS has a solution if and only if there exist $P_{i}>0, M_{i}, N_{i}, i \in \mathbb{V}, S>0, L$, and a scalar $\alpha>0$ such that, for each $i \in \mathbb{V}$,

$$
\begin{aligned}
& \Pi_{i}\left(\alpha, M_{i}, N_{i}\right) \\
\triangleq & \left(\mathcal{Z}_{i}^{\perp}\right)^{T}\left[\begin{array}{ccc}
\Pi_{1 i}\left(\alpha, M_{i}, N_{i}\right) & \# & \# \\
\Pi_{2 i} & -2 \mathcal{S}_{+}(\alpha) & \# \\
\mathcal{C}_{i} & 0 & -\gamma_{i} I
\end{array}\right] \mathcal{Z}_{i}^{\perp} \\
< & 0
\end{aligned}
$$

where

$$
\begin{aligned}
\Pi_{1 i}\left(\alpha, M_{i}, N_{i}\right)= & 2 \alpha \Upsilon_{i}-2 \alpha \operatorname{Herm}\left(\mathcal{C}_{y i}^{T} L^{T} \mathcal{M}_{i}\right) \\
& +2 \alpha \mathcal{M}_{i}^{T} S \mathcal{M}_{i}+\Lambda_{i}, \\
\Pi_{2 i}= & {\left[\begin{array}{ccc}
P_{i} & 0 & 0 \\
L \bar{C}_{y i} & -S & L \bar{D}_{y w i} \\
P_{i} & 0 & 0 \\
0 & 0 & L \bar{D}_{y w i}
\end{array}\right], } \\
\Upsilon_{i}= & {\left[\begin{array}{ccc}
0 & \bar{C}_{y i}^{T} L^{T} & 0 \\
L \bar{C}_{y i} & -S & L \bar{D}_{y w i} \\
0 & \bar{D}_{y w i}^{T} L^{T} & 0
\end{array}\right], } \\
\mathcal{M}_{i}= & {\left[\begin{array}{lll}
M_{i} & 0 & N_{i}
\end{array}\right], } \\
\mathcal{C}_{y i}= & {\left[\begin{array}{lll}
\bar{C}_{y i} & 0 & \bar{D}_{y w i}
\end{array}\right] . }
\end{aligned}
$$

Under the condition, a desired control law can be obtained as

$$
K=S^{-1} L .
$$

Proof: According to Theorem 1, it suffices to prove that (12) is equivalent to (6).

(Sufficiency) $S>0$ implies that (13) is meaningful and $L=S K$. Substituting this into (12), and noting, for any $M_{i}$ and $N_{i},-\mathcal{C}_{y i}^{T} K^{T} S K \mathcal{C}_{y i} \leq-\operatorname{Herm}\left(\mathcal{C}_{y i}^{T} K^{T} S^{T} \mathcal{M}_{i}\right)+$ $\mathcal{M}_{i}^{T} S \mathcal{M}_{i}$, one can easily verify that (6) holds.

(Necessity) Assume that (6) holds. Then, by setting $M_{i}=$ $K \bar{C}_{y i}$ and $N_{i}=K \bar{D}_{y w i}$, one has that

$$
\begin{aligned}
& -\mathcal{C}_{y i}^{T} K^{T} S K \mathcal{C}_{y i} \\
= & -\mathcal{C}_{y i}^{T} K^{T} S K \mathcal{C}_{y i}+\left(\mathcal{M}_{i}-K \mathcal{C}_{y i}\right)^{T} S\left(\mathcal{M}_{i}-K \mathcal{C}_{y i}\right) \\
= & -\operatorname{Herm}\left(\mathcal{C}_{y i}^{T} K^{T} S^{T} \mathcal{M}_{i}\right)+\mathcal{M}_{i}^{T} S \mathcal{M}_{i} .
\end{aligned}
$$

Substituting this into (6) and letting $L=S K$ yield that (12) holds. This completes the proof.
When $\alpha, M_{i}$, and $N_{i}$ are fixed, (12) becomes a strict LMI, which could be verified easily by conventional LMI solver. According to the proof of Theorem 1, the larger the $\alpha$, the higher the reduction in the conservatism of (12). If (12) does not hold for a sufficiently large $\alpha>0$, it would be likely to conclude that Problem SHS has no solution. Hence, $\alpha$ can be set to be a large value. The remaining problem is how to select $M_{i}$ and $N_{i}$. It can be seen from the proof of Theorem 2 that the scalar $\epsilon$ satisfying $\Pi_{i}\left(\alpha, M_{i}, N_{i}\right)<\epsilon I$ achieves its minimum when $M_{i}=K \bar{C}_{y i}$ and $N_{i}=K \bar{D}_{y w i}$, which can be used to construct an iteration rule. The following proposition gives a refined characterization on $\alpha, M_{i}$, and $N_{i}$.

Proposition 1: When other variables, that is, $P_{i}>0, S>$ 0 , and $L$, are fixed, the following relationship holds for any $M_{i}, N_{i}, i \in \mathbb{V}$, and $\alpha_{M}>\alpha_{m}>0$,

$$
\begin{aligned}
& \Pi_{i}\left(\alpha_{M}, S^{-1} L \bar{C}_{y i}, S^{-1} L \bar{D}_{y w i}\right) \\
\leq & \Pi_{i}\left(\alpha_{m}, S^{-1} L \bar{C}_{y i}, S^{-1} L \bar{D}_{y w i}\right) \\
\leq & \Pi_{i}\left(\alpha_{m}, M_{i}, N_{i}\right) .
\end{aligned}
$$

Proof: The second " $\leq$ " follows immediately from the proof of Theorem 2. As for the first " $\leq$ ", it suffices to show $-\mathcal{S}_{+}\left(\alpha_{M}\right) \leq$ $-\mathcal{S}_{+}\left(\alpha_{m}\right)$ and $\Pi_{1 i}\left(\alpha_{M}, S^{-1} L \bar{C}_{y i}, S^{-1} L \bar{D}_{y w i}\right) \leq$ $\Pi_{1 i}\left(\alpha_{m}, S^{-1} L \bar{C}_{y i}, S^{-1} L \bar{D}_{y w i}\right)$. The former one is obvious, and the latter one can be verified by noting that

$$
\begin{aligned}
& \Pi_{1 i}\left(\alpha_{M}, S^{-1} L \bar{C}_{y i}, S^{-1} L \bar{D}_{y w i}\right) \\
& -\Pi_{1 i}\left(\alpha_{m}, S^{-1} L \bar{C}_{y i}, S^{-1} L \bar{D}_{y w i}\right) \\
= & 2\left(\alpha_{m}-\alpha_{M}\right)\left[\begin{array}{c}
-\bar{C}_{y i}^{T} L^{T} \\
S \\
-\bar{D}_{y w i}^{T} L^{T}
\end{array}\right] S^{-1}\left[\begin{array}{c}
-\bar{C}_{y i}^{T} L^{T} \\
S \\
-\bar{D}_{y w i}^{T} L^{T}
\end{array}\right]^{T} \\
\leq & 0 .
\end{aligned}
$$

This completes the proof.

From the proposition, it can be further revealed that the scalar $\epsilon$ satisfying $\Pi_{i}\left(\alpha, M_{i}, N_{i}\right)<\epsilon I$ achieves its global minimum only if $\alpha \rightarrow+\infty, M_{i}=S^{-1} L \bar{C}_{y i}=K \bar{C}_{y i}$, and $N_{i}=S^{-1} L \bar{D}_{y w i}=K \bar{D}_{y w i}$. In view of this and aforementioned analysis, the following iterative algorithm is constructed to solve the condition of Theorem 2 .

\section{Algorithm 1:}

1) (Initialization) Set $\nu=1$ and $\alpha$ to be a sufficiently large value (for example, $\alpha=10^{4}$ ). Select initial values $M_{i}^{(\nu)}$ and $N_{i}^{(\nu)}, i \in \mathbb{V}$, such that every system in the following family with $\bar{u}_{i}(t)=M_{i}^{(\nu)} x_{c l i}(t)+$ $N_{i}^{(\nu)} w_{i}(t)$ is asymptotically stable with $\left\|\mathcal{T}_{z_{i} w_{i}}^{\text {aux }}\right\|_{\infty}<$ $\gamma_{\infty i}$, where $\mathcal{T}_{z_{i} w_{i}}^{\text {aux }}(s)$ represents the corresponding closed-loop transfer function matrix of the $i$ th system from $w_{i}$ to $z_{i}$.

$$
\left\{\begin{aligned}
\dot{x}_{c l i}(t) & =\bar{A}_{i} x_{c l i}(t)+\bar{B}_{i} \bar{u}_{i}(t)+\bar{B}_{w i} w_{i}(t), \\
z_{i}(t) & =\bar{C}_{i} x_{c l i}(t)+\bar{D}_{i} \bar{u}_{i}(t)+\bar{D}_{w i} w_{i}(t) .
\end{aligned}\right.
$$

Set $\epsilon_{*}^{(\nu)}>0$ to be a large number and $c$ to be an arbitrary positive scalar. 
2) (Iteration) For fixed $\alpha, M_{i}^{(\nu)}$, and $N_{i}^{(\nu)}$, solve the following sequential optimization problem:

Minimize $\epsilon$ subject to, for each $i \in \mathbb{V}, P_{i}>0, S>0$, and

$$
\begin{array}{r}
\Pi_{i}\left(\alpha, M_{i}^{(\nu)}, N_{i}^{(\nu)}\right)<\epsilon I \\
\epsilon \geq-c
\end{array}
$$

Denote $\epsilon_{*}^{(\nu+1)}, S^{(\nu)}$, and $L^{(\nu)}$ as the optimal value of $\epsilon, S$, and $L$, respectively.

3) (Criterion) If $\epsilon_{*}^{(\nu+1)}<0$, then there exists a solution to Problem SHS, and a control law can be obtained as (13), that is, $K=\left(S^{(\nu)}\right)^{-1} L^{(\nu)}$. STOP.

Else if $\left|\epsilon_{*}^{(\nu+1)}-\epsilon_{*}^{(\nu)}\right| \leq \delta$, where $\delta$ is a prescribed tolerance, then go to Step 4, else update

$$
\begin{aligned}
M_{i}^{(\nu+1)} & =\left(S^{(\nu)}\right)^{-1} L^{(\nu)} \bar{C}_{y i} \\
N_{i}^{(\nu+1)} & =\left(S^{(\nu)}\right)^{-1} L^{(\nu)} \bar{D}_{y w i}
\end{aligned}
$$

and set $\nu=\nu+1$, then go to Step 2 .

4) (Termination) There may not exist a solution to Problem SHS. STOP (or choose other $\alpha$ and initial values $M_{i}^{(1)}, N_{i}^{(1)}$, then run the algorithm again).

Remark 4: It can be seen easily from Proposition 1 and (16) that the sequence $\epsilon_{*}^{(\nu)}$ is monotonic decreasing with respect to $\nu$, that is, $\epsilon_{*}^{(\nu)} \leq \epsilon_{*}^{(\nu-1)}$, and bounded from below by $-c$. Therefore, the convergence of the iteration is guaranteed.

Remark 5: The initial values $M_{i}^{(1)}$ and $N_{i}^{(1)}$ are the "state-feedback $\mathcal{H}_{\infty}$ matrices", which can be determined by existing approaches. If no such matrices are found, it can be concluded immediately that Problem SHS has no solution. Like many other iterative algorithms, the sequence of iterates depends on the selection of initial values, and appropriate selection of $M_{i}^{(1)}$ and $N_{i}^{(1)}$ will improve the solvability. In addition, various search routines, such as fminsearch.m provided in MATLAB Optimization Toolbox, can be applied to find the optimal $\alpha$ for each iteration, if one can afford more computational effort. In summary, the global optimality of the iteration is affected by the initial values and the tuning parameter $\alpha$, and is thus generally not guaranteed. Further improvement will be discussed in the next subsection

\section{Desirable Initial Value}

As mentioned previously, the initial values $M_{i}^{(1)}, N_{i}^{(1)}$, and the tuning parameter $\alpha$ may affect the global optimality of the iteration. To see this in a detailed way, let us consider

$$
\begin{aligned}
& -\alpha \mathcal{C}_{y i}^{T} K^{T} S K \mathcal{C}_{y i} \\
\leq & -\alpha \operatorname{Herm}\left(\mathcal{C}_{y i}^{T} K^{T} S^{T} \mathcal{M}_{i}\right)+\alpha \mathcal{M}_{i}^{T} S \mathcal{M}_{i} \\
= & -\alpha \mathcal{C}_{y i}^{T} K^{T} S K \mathcal{C}_{y i}+\alpha\left(\mathcal{M}_{i}-K \mathcal{C}_{y i}\right)^{T} S\left(\mathcal{M}_{i}-K \mathcal{C}_{y i}\right) .
\end{aligned}
$$

It follows from this inequality that if Problem SHS has a solution $K^{*}$, then (12) will also be feasible, provided that $\left\|\alpha\left(\mathcal{M}_{i}-K^{*} \mathcal{C}_{y i}\right)^{T} S\left(\mathcal{M}_{i}-K^{*} \mathcal{C}_{y i}\right)\right\|$ is sufficiently small. The converse is also true. In view of this, it is natural to improve the solvability of the iterative calculation by reducing $\left\|\alpha\left(\mathcal{M}_{i}-K^{*} \mathcal{C}_{y i}\right)^{T} S\left(\mathcal{M}_{i}-K^{*} \mathcal{C}_{y i}\right)\right\|$, which can be achieved through adjusting the two parameters $\alpha$ and $\mathcal{M}_{i}$, namely, making $\alpha$ and $\left\|\mathcal{M}_{i}-K^{*} \mathcal{C}_{y i}\right\|$ sufficiently small. From Proposition 1, however, $\alpha$ should be large in order to achieve global optimality of the condition in Theorem 2. Hence, the only way is to reduce $\left\|\mathcal{M}_{i}-K^{*} \mathcal{C}_{y i}\right\|$ by choosing appropriate $\mathcal{M}_{i}$. Since

$$
\begin{aligned}
\mathcal{M}_{i}-K^{*} \mathcal{C}_{y i} & =\left(\mathbf{M}_{i}-K^{*} \mathbf{C}_{y i}\right)\left[\begin{array}{lll}
I & 0 & 0 \\
0 & 0 & I
\end{array}\right], \\
\mathbf{M}_{i} & =\left[\begin{array}{ll}
M_{i} & N_{i}
\end{array}\right] \\
\mathbf{C}_{y i} & =\left[\begin{array}{ll}
\bar{C}_{y i} & \bar{D}_{y w i}
\end{array}\right]
\end{aligned}
$$

it suffices to reduce $\left\|\mathbf{M}_{i}-K^{*} \mathbf{C}_{y i}\right\|$. To this end, the following theorem, which plays a central role in selecting $\mathbf{M}_{i}$, is provided.

Theorem 3: For some matrices $\mathbf{M}_{i}$ and scalars $\gamma_{i}>0$, $i \in \mathbb{V}$, the following two statements are equivalent

1) Problem SHS has a solution $K^{*}$ satisfying $\left\|\mathbf{M}-K^{*} \mathbf{C}_{y}\right\| \leq \mu_{1}$, where $\mu_{1}>0$ is a sufficiently small scalar.

2) $\mathbf{M}_{i} \in \mathbb{S}_{\infty i}, i \in \mathbb{V}$, and $\left\|\mathbf{M} \mathbf{C}_{y}^{\perp}\right\| \leq \mu_{2}$, where $\mu_{2}>0$ is a sufficiently small scalar, and

$$
\begin{gathered}
\mathbb{S}_{\infty i}=\left\{\mathbf{M}_{i} \mid \text { the } i\right. \text { th system of (14) with } \\
\bar{u}_{i}(t)=M_{i} x_{c l i}(t)+N_{i} w_{i}(t)
\end{gathered}
$$

is asymptotically stable with

$$
\begin{aligned}
& \left.\left\|\mathcal{T}_{z_{i} w_{i}}^{a u x}\right\|_{\infty}<\gamma_{\infty i}\right\} \\
& \mathbf{M}=\left[\begin{array}{llll}
\mathbf{M}_{1} & \mathbf{M}_{2} & \cdots & \mathbf{M}_{r}
\end{array}\right], \\
& \mathbf{C}_{y}=\left[\begin{array}{llll}
\mathbf{C}_{y 1} & \mathbf{C}_{y 2} & \cdots & \mathbf{C}_{y r}
\end{array}\right] .
\end{aligned}
$$

Proof: 1) $\Rightarrow 2$ ) It follows from 1) that $\mathbf{M}_{i}=K^{*} \mathbf{C}_{y i}+$ $\Sigma_{i}$, where $\Sigma_{i}$ is a sufficiently small perturbation, and the $i$ th system of (14) with $\bar{u}_{i}(t)=M_{i} x_{c l i}(t)+N_{i} w_{i}(t)=$ $K^{*} \mathbf{C}_{y i}\left[\begin{array}{ll}x_{c l i}^{T}(k) & w^{T}(k)\end{array}\right]^{T}+\Sigma_{i}\left[\begin{array}{cc}x_{c l i}^{T}(k) & w^{T}(k)\end{array}\right]^{T}$ is still asymptotically stable with $\left\|\mathcal{T}_{z_{i} w_{i}}^{\text {aux }}\right\|_{\infty}<\gamma_{\infty i}$, which means $\mathbf{M}_{i} \in \mathbb{S}_{\infty i}$. In addition, $\left\|\mathbf{M C}_{y}^{\perp}\right\|=$ $\left\|\left(\mathbf{M}-K^{*} \mathbf{C}_{y}\right) \mathbf{C}_{y}^{\perp}\right\| \leq \mu_{1}\left\|\mathbf{C}_{y}^{\perp}\right\| \triangleq \mu_{2}$, which is sufficiently small.

$2) \Rightarrow 1$ ) It is noted that if $\operatorname{rank}\left(\mathbf{C}_{y}\right)=n_{1}<n_{y}+n_{c}$, then $\mathbf{C}_{y}$ can be QR-factorized as

$$
\mathbf{C}_{y}=\mathbf{U}\left[\begin{array}{c}
\mathbf{C}_{1} \\
0
\end{array}\right]
$$

where $\mathbf{U} \in \mathbb{R}^{\left(n_{y}+n_{c}\right) \times\left(n_{y}+n_{c}\right)}$ is an orthogonal matrix, $\mathbf{C}_{1} \in$ $\mathbb{R}^{n_{1} \times r\left(n+n_{c}+n_{w}\right)}$ is a matrix with full row rank, and

$$
\mathbf{C}_{1}^{\perp}=\mathbf{C}_{y}^{\perp} .
$$

Now define $K^{*}$ as

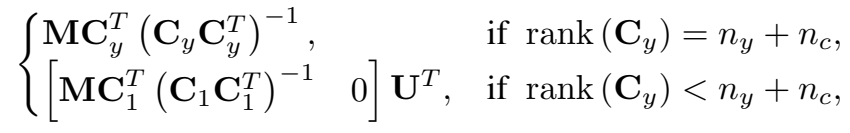


which implies that

$$
\begin{cases}\mathbf{M C}_{y}^{T}-K^{*} \mathbf{C}_{y} \mathbf{C}_{y}^{T}=\mathbf{0}, & \text { if } \operatorname{rank}\left(\mathbf{C}_{y}\right)=n_{y}+n_{c}, \\ \mathbf{M C}_{1}^{T}-K^{*} \mathbf{C}_{y} \mathbf{C}_{1}^{T}=\mathbf{0}, & \text { if } \operatorname{rank}\left(\mathbf{C}_{y}\right)<n_{y}+n_{c}\end{cases}
$$

With this and (17), one obtains that

$$
\begin{aligned}
\left(\mathbf{M}-K^{*} \mathbf{C}_{y}\right) \times\left[\begin{array}{ll}
\mathbf{C}_{y}^{T} & \mathbf{C}_{y}^{\perp}
\end{array}\right] & =\left[\begin{array}{ll}
0 & \mathbf{M} \mathbf{C}_{y}^{\perp}
\end{array}\right], \\
\text { if } \operatorname{rank}\left(\mathbf{C}_{y}\right) & =n_{y}+n_{c}, \\
\left(\mathbf{M}-K^{*} \mathbf{C}_{y}\right) \times\left[\begin{array}{ll}
\mathbf{C}_{1}^{T} & \mathbf{C}_{1}^{\perp}
\end{array}\right] & =\left[\begin{array}{ll}
0 & \mathbf{M} \mathbf{C}_{y}^{\perp}
\end{array}\right], \\
\text { if } \operatorname{rank}\left(\mathbf{C}_{y}\right) & <n_{y}+n_{c},
\end{aligned}
$$

which, by noting the invertibility of $\left[\begin{array}{ll}\mathbf{C}_{y}^{T} & \mathbf{C}_{y}^{\perp}\end{array}\right]$ and $\left[\begin{array}{ll}\mathbf{C}_{1}^{T} & \mathbf{C}_{1}^{\perp}\end{array}\right]$, implies that

$$
\begin{aligned}
& \left\|\mathbf{M}-K^{*} \mathbf{C}_{y}\right\| \\
\leq & \mu_{2} \max \left\{\left\|\left[\begin{array}{ll}
\mathbf{C}_{y}^{T} & \mathbf{C}_{y}^{\perp}
\end{array}\right]^{-1}\right\|,\left\|\left[\begin{array}{ll}
\mathbf{C}_{1}^{T} & \mathbf{C}_{1}^{\perp}
\end{array}\right]^{-1}\right\|\right\} \\
\triangleq & \mu_{1},
\end{aligned}
$$

which is sufficiently small. Similar to the derivation in $1) \Rightarrow 2$ ), one further obtains that $K^{*}$ is a solution to Problem SHS.

From this theorem, one may conclude that desirable initial values should be in $\mathbb{S}_{\infty i}$ and $\left\|\mathbf{M C}_{y}^{\perp}\right\|$ should be small enough. For the extreme case that $\mathbf{M}_{i} \in \mathbb{S}_{\infty i}$ and $\left\|\mathbf{M C} \mathbf{C}_{y}^{\perp}\right\|=0$, (12) must be feasible for a sufficiently large scalar $\alpha>0$. Based on this, some optimization techniques such as D-K type iteration [21] or ellipsoidal approximation could be developed to find a desirable initial value. Details are omitted here due to page length consideration.

\section{Extension to SHS with Structural Gains or Strong Stabi- lizability}

Theorem 4: Problem SHS has a structural solution if and only if there exist $P_{i}>0, M_{i}, N_{i}, i \in \mathbb{V}$, diagonal $S>0$, $L$ with a prescribed pattern, and a scalar $\alpha>0$ such that, for each $i \in \mathbb{V}$, (12) holds.

Proof: Noting that $S$ can be set to any positive definite matrix and $S^{-1} L$ has the same structure with $L$, the proof follows immediately.

Theorem 5: Problem SHS has a $\gamma_{K}$-stable solution if and only if there exist $P_{i}>0, M_{i}, N_{i}, i \in \mathbb{V}, S=$ $\operatorname{diag}\left(P_{K}, I\right)>0, L=\left[\begin{array}{ll}L_{1} & L_{2} \\ L_{3} & L_{4}\end{array}\right]$ with the partition compatible with $K$, and a scalar $\alpha>0$ such that, for each $i \in \mathbb{V}$, (12) and the following LMI hold:

$$
\left[\begin{array}{ccc}
L_{1}+L_{1}^{T} & \# & \# \\
L_{2}^{T} & -\gamma_{K} I & \# \\
L_{3} & L_{4} & -\gamma_{K} I
\end{array}\right]<0
$$

Proof: Noting that the structure of $S$ and applying the bounded real lemma to the controller in (2), the proof follows immediately.

\section{CONCLUSIONS}

In this paper, a novel necessary and sufficient condition for simultaneous $\mathcal{H}_{\infty}$ stabilizability has been established in terms of matrix inequalities with a free parameterization matrix. A monotonic structure of the condition enables one to solve it via an convergent iterative algorithm, and a special property of feasible solutions provides a possibility to improve the solvability via simple optimization on the initial values. In addition, additional design specifications such as structural controller gains or strong stabilizability can be readily incorporated into the design procedure.

\section{REFERENCES}

[1] R. Saeks and J. Murray, "Fractional representation, algebraicgeometry, and the simultaneous stabilization problem," IEEE Trans. Automatic Control, vol. 27, no. 4, pp. 895-903, Aug. 1982.

[2] M. Vidyasagar and N. Viswanadham, "Algebraic design techniques for reliable stabilization," IEEE Trans. Automatic Control, vol. 27, no. 5, pp. 1085-1095, Oct. 1982.

[3] Y. Cao and J. Lam, "On simultaneous $\mathcal{H}_{\infty}$ control and strong $\mathcal{H}_{\infty}$ stabilizations," Automatica, vol. 36, no. 6, pp. 859-865, Jun. 2000.

[4] P. D. Olivier, "On the relationship between the model order reduction problem and the simultaneous stabilization problem," IEEE Trans. Automatic Control, vol. AC-32, no. 1, pp. 54-55, Jan. 1987.

[5] T. E. Djaferis, "To stabilize a $k$ real parameter affine family of plants it suffices to simultaneously stabilize $4^{k}$ polynomials," Syst. Control Lett., vol. 16, no. 3, pp. 187-193, Mar. 1991.

[6] A.-W. Saif, D.-W. Gu, D. Kavranoglu, and I. Postlethwaite, "Simultaneous stabilization of MIMO systems via robustly stabilizing a central plant," IEEE Trans. Automatic Control, vol. 47, no. 2, pp. 363-369, Feb. 2002.

[7] F. J. Arteaga-Bravo and G. O. Beale, "Simultaneous stabilization with multiple bounded domains of stability in chemical process control," in Proceedings of The 1995 IEEE IECON-21st International Conference on Industrial Electronics, Control, and Instrumentation, vol. 1/2, 1995 , pp. 830-835.

[8] J. Stoustrup and V. D. Blondel, "Fault tolerant control: A simultaneous stabilization result," IEEE Trans. Automatic Control, vol. 49, no. 2, pp. 305-310, Feb. 2004.

[9] E. Emre, "Simultaneous stabilization with fixed closed-loop characteristic polynomial," IEEE Trans. Automatic Control, vol. AC-28, no. 1, pp. 103-104, Jan. 1983.

[10] C. A. Desoer and C. A. Lin, "Simultaneous stabilization of nonlinear systems," IEEE Trans. Automatic Control, vol. AC-29, no. 5, pp. 455457, May 1984.

[11] W. E. Schmitendorf and C. V. Hollot, "Simultaneous stabilization via linear state feedback control," IEEE Trans. Automatic Control, vol. 34, no. 9, pp. 1001-1005, Sep. 1989.

[12] V. D. Blondel and J. N. Tsitsiklis, "A survey of computational complexity results in systems and control," Automatica, vol. 36, no. 9, pp. 1249-1274, Sep. 2000.

[13] - "NP-hardness of some linear control design problems," SIAM J. Control Optim., vol. 35, no. 6, pp. 2118-2127, Nov. 1997.

[14] V. D. Blondel and M. Gevers, "Simultaneous stabilizability of 3 linearsystems is rationally undecidable," Mathematics of Control Signals and Systems, vol. 6, no. 2, pp. 135-145, 1993.

[15] D. Henrion, S. Tarbouriecha, and M. Šebek, "Rank-one LMI approach to simultaneous stabilization of linear systems," Syst. Control Lett., vol. 38, no. 2, pp. 79-89, Oct. 1999.

[16] Y. Cao, Y. Sun, and J. Lam, "Simultaneous stabilization via static output feedback and state feedback," IEEE Trans. Automatic Control, vol. 44, no. 6, pp. 1277-1282, Jun. 1999.

[17] D. E. Miller and T. Chen, "Simultaneous stabilization with nearoptimal $\mathcal{H}_{\infty}$ performance," IEEE Trans. Automatic Control, vol. 47, no. 12, pp. 1986-1998, Dec. 2002.

[18] P. H. Lee and Y. C. Soh, "Synthesis of simultaneous stabilizing $\mathcal{H}_{\infty}$ controller," Int. J. Control, vol. 78, no. 18, pp. 1437-1446, Dec. 2005.

[19] S. Boyd, L. El Ghaoui, E. Feron, and V. Balakrishnan, Linear Matrix Inequalities in System and Control Theory. Philadelphia: Society for Industrial and Applied Mathematics, 1994.

[20] K. Zhou and J. C. Doyle, Essentails of Robust Control. Upper Saddle River, N.J.: Prentice Hall, 1998.

[21] M. A. Rotea and T. Iwasaki, "An alternative to the D-K iteration?" in Proceedings of American Control Conference, vol. 1, Jun. 1994, pp. 53-57. 